\title{
Tratamiento de la imagen corporal en los trastornos alimentarios y cambio clínicamente significativo
}

\author{
José H. Marco ${ }^{1 *}$, Conxa Perpiñá2 y Cristina Botella ${ }^{3}$ \\ 1 Universidad Católica de Valencia "San Vicente Mártir" \\ 2 Universidad de Valencia. \\ ${ }^{3}$ Universidad Jaume I de Castellón
}

\begin{abstract}
Resumen: Las alteraciones de la imagen corporal son un factor mantenedor y de pronóstico en los trastornos alimentarios. Los tratamientos actuales para los trastornos alimentarios se podrían beneficiar de la intervención directa sobre la imagen corporal. En el siguiente trabajo se realiza un estudio controlado en el que se comprara la Terapia Cognitivo Conductual para los trastornos alimentarios con y sin un componente para el tratamiento de la imagen corporal apoyado con técnicas de realidad virtual. El objetivo de este trabajo fue comprobar si ambos tipos de tratamiento producen un cambio clínicamente significativo al finalizar el tratamiento y en el seguimiento al año en la imagen corporal, en la psicopatología especifica de los trastornos alimentarios y en la psicopatología general. Para ello evaluamos y tratamos a 34 participantes diagnosticadas de trastorno alimentario y comparamos sus resultados con un grupo de mujeres de población general con baja vulnerabilidad a los trastornos alimentarios. Los resultados indican que después del tratamiento se ha producido un cambio fiable y clínicamente significativo únicamente en la condición en la que se ha intervenido en la imagen corporal. Las implicaciones de este resultado y sus limitaciones son comentadas.

Palabras claves: realidad virtual; cambio clínicamente significativo, bulimia nerviosa, imagen corporal, trastornos alimentarios, tratamiento psicológico, terapia cognitivo conductual.
\end{abstract}

\section{Introducción}

Las alteraciones de la imagen corporal (IC) son una de las características clínicas de mayor importancia en los trastornos alimentarios (Bully, Elosua y Lopez-Jauregui, 2012; Garner, 2002; Nye y Cash, 2006; Schwartz y Browell, 2004; Stice, 2002). La insatisfacción con el cuerpo y las alteraciones de la IC se encuentran asociadas con conductas y actitudes problemáticas hacia la comida, como es la falta de control sobre la comida, hacer dietas restrictivas y la existencia de sintomatología bulímica. Por este motivo, la persistencia de la insatisfacción corporal después del tratamiento de los trastornos alimentarios es un predictor fiable de recaída en la bulimia nerviosa (Cash y Hrabrosky, 2004; Shisslak y Crago, 2001; Stice, 2002). La IC es uno de los factores de pronóstico más importantes, en el tratamiento de la bulimia nerviosa (BN) (Fairburn, Peveler, Jones, Hope y Doll, 1993; Stice y Shaw, 2002) como de la anorexia nerviosa (AN) (Gleaves, Williamson y Barker, 1993; Thompson, 1992).

Pese a la importancia de la IC, en las revisiones de los tratamientos para los trastornos alimentarios (Farrell, Shafran y Lee, 2006) se observa que la mayoría de estos no evalúan ni tratan la IC y, en estudios en los que la IC es un

* Dirección para correspondencia [Correspondence address]: José Heliodoro Marco. Departamento de Personalidad, Evaluación y Tratamientos en ciencias de la Salud. Facultad de Psicología y Ciencias de la Salud. Universidad Católica de Valencia "San Vicente Mártir". C/ Guillem de Castro, 175, 46008 Valencia (España).

E-mail: joseheliodoro.marco@ucv.es
Title: The treatment of the body image disturbances in eating disorders and clinically significant change.

Abstract: Body image disturbance is a significant maintenance and prognosis factor in eating disorders. Hence, existing eating disorder treatments can benefit from direct intervention in patients' body image. This paper includes a controlled study comparing cognitive behavioural treatment for eating disorders with and without a component for body image treatment using virtual reality techniques. The objective of this work was to check if both types of treatment produced a change clinically significant at the end of the treatment and the follow-up to the year, in body image, eating disorders and general psychopathology. Thirty-four participants diagnosed with eating disorders were treated and compared with the general population with low vulnerability to eating disorders. Results showed that only in the patients who received the body image treatment obtained a change clinically significant. The implications and limitations of these results are discussed below.

Keywords: Virtual reality; clinically Significant change; anorexia nervosa; bulimia nervosa; body image; eating disorders; psychological treatment; cognitive-behavior therapy.

objetivo de tratamiento, no se evalúa cómo afecta el tratamiento realizado en la IC de las pacientes. Los tratamientos psicoeducativos para la $\mathrm{BN}$, los tratamientos puramente conductuales, los tratamientos farmacológicos, la terapia interpersonal y la terapia psicodinámica no son eficaces para la mejora global de la IC (Cash y Grant, 1996). Algunos tratamientos para los trastornos alimentarios (Thompson, Heimberg y Clark, 1996) incluyen componentes educativos dirigidos a la IC, en la BN (Fairburn, 2002) y en el caso de la AN (Vitousek, 2002) sin embargo se desconoce el efecto de la intervención sobre la IC de estos programas (Nye y Cash, 2006). Por tanto, parece necesaria la realización de estudios comparativos entre tratamientos con y sin un componente centrado en la IC donde se pueda comprobar la eficacia estadística y clínica del tratamiento.

Las intervenciones centradas en la alteraciones de la IC, pueden mejorar los Tratamientos Empíricamente Validados para los trastornos alimentarios (NICE, 2004). Para ello es necesario diseñar programas de tratamiento para los trastornos alimentarios que incorporen componentes para el tratamiento de la IC (Cash y Hrabrosky, 2004; Farrell et al., 2006). Desde una perspectiva transdiagnóstica (Fairburn, Cooper y Shafran, 2003) es necesario la intervención en la IC para prevenir el mantenimiento de la psicopatología de los trastornos alimentarios. Algunos avances se han realizado en esta línea incorporando nuevos componentes a los tratamientos existentes, tales como la Terapia Cognitivo Conductual (TCC) para la BN (Fairburn, Marcus y Wilson, 1993) en su versión ampliada (Fairburn et al.. 2009). Sin 
embargo, hasta el momento no se ha realizado un estudio controlado donde se compruebe la eficacia diferencial de la intervención de la IC en los trastornos alimentarios (Nye y Cash, 2006). En este sentido, puede ser interesante la realización de estudios comparativos entre tratamientos con y sin un componente centrado en la IC en los que se pueda comprobar la eficacia de los tratamientos.

Los estudios de eficacia entre tratamientos suelen realizar comparaciones estadísticas antes y después del tratamiento, de manera que podemos concluir si una condición de tratamiento ha mejorado más que otra condición. Sin embargo, cada vez más autores (Jacobson y Truax, 1991; Lundgren, Danoff-Burg y Anderson, 2004; Ogles, Lunnen y Bonesteel, 2001; Sheldrick, Kendall y Heimberg, 2001) sugieren que cuando se trata de analizar la eficacia de tratamientos, no nos podemos limitar al uso de comparaciones estadísticas que sólo indican si una condición cambia estadísticamente más que la otra, ya que este tipo de comparaciones no nos proporciona información sobre si este cambio implica una mejoría clínicamente significativa en los participantes tras el tratamiento. El cambio clínicamente significativo (Jacobson y Truax, 1991) se produce al comprobar que en el paciente se ha producido un cambio que le lleva a la recuperación del estado normal de salud, en otras palabras, cuando la sintomatología que presenta el paciente es similar a la que presenta la población general.

Para comprobar si se ha producido un cambio clínicamente significativo, algunos autores (p. ej., Follet y Callaghan, 2001; Kendall, Marrs-García, Nath y Sheldrick, 1999) sugieren realizar dos tipos de análisis: en primer lugar, mediante el Índice de Cambio Fiable (ICF) (Christensen y Mendoza, 1986; Jacobson, Follette y Revenstorf, 1986; Jacobson y Revestorf, 1988) que se trata de un análisis de la fiabilidad del cambio producido por el tratamiento, de manera que se compruebe que los cambios, antes y después del mismo no son debidos a un error de la medida y, por tanto, se deben a la influencia de la intervención; en segundo lugar, mediante un test de equivalencia, a través del cual se comprueba si después de un tratamiento los participantes presentan puntuaciones que se encuentran dentro del rango normal para su grupo (Lungreen, et al., 2004).

En las últimas décadas se ha utilizado el análisis del cambio clínicamente significativo para comprobar la eficacia del tratamiento de los trastornos de conducta (Sheldrick, et al., 2001), del estrés marital (Jacobson et al., 1984), de las cefaleas tensionales (Blanchard et al., 1990). En el caso concreto de los trastornos alimentarios, existen muy pocos estudios donde se haya realizado un análisis de la eficacia clínica de los tratamientos aplicados. En un meta-análisis (Lundgreen, et al., 2004) concluyeron que la TCC para la BN producía cambios clínicamente significativos en la reducción de atracones y en la de purgas, pero no en las alteraciones de la IC, ni en las actitudes y creencias sobre el peso y la comida.

En un estudio previo (Marco, Perpiñá y Botella, 2013) demostraron que la TCC para los trastornos alimentarios, ampliada con un componente específico para la IC mediante técnicas de RV fue más eficaz que la TCC para los trastornos alimentarios sin un componente de tratamiento específico para la IC. Esta mejoría fue mayor tanto desde un análisis de diferencias estadísticamente significativas como desde un análisis del cambio clínicamente significativo. Al finalizar el tratamiento y en el seguimiento al año, en la condición que se trató la IC, presentaban una reducción de la psicopatología del trastornos alimentario y de la IC a niveles similares a la de la población general. En la condición que no se ha intervenido específicamente en la IC no se produjo una mejoría clínicamente significativa.

En el presente trabajo, vamos un paso más allá, ya que hay que tener en cuenta que la población general presenta actitudes, creencias, emociones y comportamientos que están presentes en personas con trastornos alimentarios y que comparten en menor grado. Este descontento normativo (Rodin, Silberstein y Striegel-Moore, 1984) hacia el cuerpo tiene como consecuencia que el $11 \%$ de la población femenina presente actitudes, creencias y comportamientos relacionados con el cuerpo y la comida similares a los que presentan las pacientes diagnosticadas de AN y BN (Cash y Habrosky, 2004; Clarke y Palmer, 1983). Por lo tanto, para comprobar que un tratamiento dirigido a participantes diagnosticadas de trastornos alimentarios ha producido una mejoría clínicamente significativa los resultados tendrían que ser comparados con la población general con baja vulnerabilidad a padecer un trastorno alimentario.

El objetivo de este trabajo fue comprobar si añadir un componente de tratamiento específico para la IC en la TCC para los trastornos alimentarios, produce una mejoría mayor que la TCC para los trastornos alimentarios estándar. Para ello comprobaremos si ambas condiciones de tratamiento son capaces de producir un cambio clínicamente significativo, mediante comparación con la población general con baja vulnerabilidad a tener un trastorno alimentario. Más específicamente, se quiso comprobar si ese cambio clínicamente significativo se produciría en las medidas de IC, en la psicopatología alimentaría, y en la psicopatología general asociada.

\section{Método}

\section{Participantes}

Las participantes provenían del Hospital Provincial de Castellón. Los criterios de inclusión fueron: pacientes diagnosticadas de un trastorno alimentario según los criterios del DSM-IV-TR (APA, 2000). Los criterios de exclusión fueron: IMC $<16$, abuso de sustancias y que existiera un manifiesto riesgo de suicidio.

La muestra, estaba compuesta por 34 pacientes, todas ellas mujeres con los siguientes diagnósticos: 17 con BN (16 tipo purgativo y 1 tipo no purgativo), 12 con Trastorno Alimentario No Especificado (TCANE) y 5 con AN (2 tipo purgativo y 3 tipo restrictivo). El rango de edad de las pacientes era muy amplio, de los 15 a los 40 años con una edad media de 21.82 (5.75). El Índice de Masa Corporal de las pa- 
cientes iba desde 16 hasta 32 con una media de 21.5(4.28) y, en cuanto a los años de evolución de la enfermedad de las pacientes, éste iba desde 1 hasta 16 años siendo la evolución media en años de 4.17 (4.1). En cuanto a la psicopatología secundaria, el $23,5 \%$ de las pacientes presentaban trastornos de la personalidad y el $32 \%$ comorbilidad con otro trastorno: el $15 \%$ cumplían criterios de un cuadro depresivo mayor, el $12 \%$ de trastorno de estrés postraumático, y el $5 \%$ trastornos de ansiedad. En cuanto al nivel educativo de las pacientes, el 29\% tenía o estaban cursando estudios universitarios, el 47\% tenía o estaba cursando estudios secundarios y el $24 \%$ tenía estudios primarios. Su participación fue voluntaria obteniéndose su consentimiento informado.

\section{Instrumentos}

Entrevista SCID I (First, Spitzer, Gibbon y Williams, 2002). Entrevista diagnóstica de trastornos mentales pertenecientes al eje I según los criterios del DSM-IV-TR (APA, 2000). Ampliamente utilizada en salud mental, ofrece buenas propiedades psicométricas: Kappa .66 (Lobbestael, Leurgans y Arntz, 2011).

Entrevista SCID-II (First, Gibbon, Spitzer, Williams y Benjamin, 1997). Entrevista de diagnóstica de trastornos mentales pertenecientes al eje II según los criterios del DSM-IV-TR (APA, 2000). Formada por 119 preguntas, presenta una fiabilidad test retest .74 para pacientes ingresados (First, Gibbon, Spitzer, Williams y Benjamin, 1999).

Body Attitud Test. BAT (Probst, Vandereycken, Van Coppenolle y Vanderlinden, 1995). Escala que evalúa la insatisfacción con el cuerpo. Está compuesta por 20 ítems con respuesta en formato Likert, que van desde 1 (nunca) hasta 5 (siempre). El rango de puntuación es de 0 hasta 100. En población española la consistencia interna es de .92 y la fiabilidad test-retest de .91 (Gila, Castro, Gómez, Toro y Salamero, 1999).

Body Image Automatic Thoughts Questionnaire. BIATQ (Cash, Lewis y Keeton, 1987). Evalúa el componente cognitivo de la IC. Está formado por 52 ítems tipo Likert que van desde 1 (nunca) hasta 5 (siempre), en los que se recogen posibles pensamientos automáticos sobre el aspecto físico. En población española (Perpiñá, Borra, Baños, Botella, Quero y Jorquera, 2003) presenta una consistencia interna de .91 en la escala general, .97 la escala negativa y .91 la escala positiva. En cuanto a la fiabilidad test-retest en población española presenta .91 para la escala general, .88 para la negativa y .76 para la positiva.

Body Areas Satisfaction Scale. BASS (Cash, 1991). Mide el grado de satisfacción e insatisfacción acerca de 10 características del cuerpo. Está compuesta por 10 ítems con respuesta tipo Likert que va desde 1 (muy insatisfecho) hasta 5 (muy satisfecho). Presenta una consistencia interna de .79 y .78 para varones y mujeres respectivamente (Cash, 1991).

Situational Inventory of Body-Image Dysphoria. SIBID (Cash, 1994). Es un instrumento que recoge las reacciones de malestar e insatisfacción con el cuerpo desencadenados por conductas o situaciones. Está compuesto por 49 ítems con respuesta tipo Likert que va desde 0 (nunca) hasta 4 (siempre). Presenta una consistencia interna en población española de .97 y fiabilidad test-retest de .86 (Perpiña, Gallego y Botella, 2006).

The Bulimic Investigatory Test, Edinburgh. BITE (Henderson y Freeman, 1987). Evalúa las características cognitivas y conductuales del trastorno por atracón y la BN. Está compuesto por 33 ítems divididos en 2 subescalas. Los ítems de la subescala Síntomas están formulados en formato dicotómico (si/no), mientas que los de la subescala Gravedadestán presentados en escala Likert (con 5 o 7 opciones en función del ítem). Presenta una fiabilidad test-retest para BN de .68. Se compone de dos escalas la de Síntomas, con una consistencia interna de .96 y la de Gravedad con una consistencia interna de .62 .

The Eating Attitudes Test. EAT-40. (Garner y Garfinkel, 1979; Garner, Olmsted, Bohr y Garfinkel, 1982). Evalúa actitudes y conductas asociadas con la AN. Está compuesto por 40 ítems organizados en 7 factores, que se contestan en una escala tipo Likert de 6 puntos. Los autores establecen un punto de corte de 30 para establecer la sintomatología clínica, por lo que en muchos estudios se utiliza esta puntuación para diferenciar en la población general a los grupos de riesgo para un trastorno alimentario. La fiabilidad test retest para un intervalo de 2-3 semanas es de .84 (Carter y Moss, 1984). En población española presenta una consistencia interna de .93 en población de AN y de .92 en BN (Castro, Toro, Salamero y Guimerá, 1991).

Brief Symptom Inventory. BSI (Derogatis y Melisaratos, 1983). Evalúa la sintomatología secundaria de las pacientes. Se compone de 49 ítems que se responden en una escala tipo Likert que va desde 0 (nada) hasta 4 (mucho). La versión española (Ruipérez, Ibañez, Lorente, Moro y Ortet, 2001) consta de 6 subescalas: Depresión, Ansiedad, Hostilidad, Somatización, Trastorno Obsesivo Compulsivo e Ideación Paranoide. Se puede obtener un Indicador General de Gravedad. La fiabilidad test-retest es de .90 (Derogatis y Melisaratos). En la versión española la consistencia interna para las escalas es de .70 a .91 (Ruipérez et al. 2001).

\section{Tratamiento}

Se establecieron dos componentes del tratamiento. El componente 1 fue la TCC para los trastornos alimentarios. Según el diagnóstico podía ser TCC para la BN (Wilson, Fairburn y Agras, 1997) y TCC para la AN (Garner, Vitousek y Pike, 1997). El componente 2 fue la TCC para las alteraciones de la IC en los trastornos alimentarios. Para este trabajo adaptamos la TCC para las alteraciones de la IC que estaban protocolizadas para la población general (Butters y Cash, 1987) a los trastornos alimentarios dividiéndola en tres fases y ampliándola a 15 sesiones de tratamiento, con las siguientes fases (Perpiñá, Botella y Baños, 2000):

Fase I. Comprende de la sesión 1 a la 3.El objetivo general de esta fase es que las pacientes sean conscientes de que 
tienen un problema de IC, que diferencien entre cuerpo e IC y aprendan que el malestar con el cuerpo no se puede eliminar cambiando el cuerpo, sino que hay que incidir en cambiar la IC. Para ello se les enseñan los principios psicoeducativos sobre que son las alteraciones de la IC, consecuencias de la IC negativa, cómo se forma la IC, qué es el peso, cómo se mantiene el peso, los trucos y engaños en la publicidad, principales factores mantenedores de la insatisfacción corporal en la sociedad actual, etc. Durante esta fase se trabajan en los escenarios de realidad virtual (índices de discrepancia de peso en relación a la ingesta; concepto de IMC y exposición a distintas constituciones corporales); durante 3 sesiones.

Fase II. Comprende de la sesión 4 a la 13. El objetivo general de esta fase es el cambio de actitudes y creencias sobre el cuerpo y la apariencia y el cambio de las conductas de evitación y seguridad que están manteniendo el problema de IC. Para ello se explican los principios fundamentales de la TCC adaptado a los problemas de IC. Se intenta que la personas dejen de evitar las situaciones sociales y del cuerpo y se aprende a afrontarlas de una forma adaptativa. Se realiza una exposición gradual al cuerpo vestido y desnudo. Se establece una nueva relación positiva con el cuerpo, produciéndose un incremento de actividades reforzantes con el mismo y el cambio de significado del cuerpo. Por último se trabaja el autoconcepto de uno mismo y la autoestima. Durante esta fase se trabajan en los escenarios de realidad virtual (construcción subjetiva del cuerpo; IC, discrepancias IC; frente al espejo) durante 5 sesiones.

Fase III, sesiones 14 y 15 . El objetivo general de la fase III, es la consolidación de los logros obtenidos en las dos fases anteriores, generando una atribución interna del resultado de tratamiento, la preparación para el alta y la prevención de recaídas.

En el componente de tratamiento de IC se aplicó un entorno de realidad virtual diseñado para este estudio. La aplicación de realidad virtual y los escenarios utilizados para el tratamiento están ampliamente descritos en varios trabajos previamente publicados (Marco et al., 2013; Perpiñá, Botella y Baños, 2003; Perpiñá et al., 2000).

\section{Diseño}

Se diseñaron dos condiciones de tratamiento En la condición de tratamiento $n^{\circ} 1$ (TTAE), se aplicó la TCC Estándar para el tratamiento de los trastornos alimentarios, esta condición está formada por el componente de tratamiento 1. En la condición de tratamiento $\mathrm{n}^{\circ} 2$ (TTAIC), se aplicó la TCC para trastornos alimentarios ampliado con un componente de tratamiento específico para la IC apoyado con técnicas de realidad virtual. En esta condición estaba formada por el componente 1 (TCC para los trastornos alimentarios) junto con el componente 2 (TCC para la IC).

Para comparar la eficacia en ambas condiciones de tratamiento se realizaron evaluaciones de las medidas en 3 momentos: antes de empezar el tratamiento, al finalizar el tratamiento y en el seguimiento al año. Para comparar la efi- cacia clínicamente significativa (Jacobson y Truax, 1991; Lundgren et al., 2004; Sheldrick et al., 2001) de ambas condiciones de tratamiento, realizamos los análisis que describo a continuación (Kendall et al., 1999). En primer lugar, comprobamos que se había producido un cambio fiable y posteriormente comparamos las diferencias de las puntuaciones clínicas a finalizar el tratamiento y en el seguimiento al año, con las puntuaciones que la población general con baja vulnerabilidad a tener un trastorno alimentario. Para ello calculamos el tamaño del efecto de las diferencias entre las puntuaciones utilizando el Índice $g$ de Hedges (Hedges y Olkin, 1985). Sólo cuando un tratamiento ha producido un cambio fiable y se ha producido una mejoría que hace que las participantes presenten puntuaciones similares a la población normal se puede considerar que es clínicamente significativo (Lundgren et al., 2004).

Para comparar los datos obtenidos en este estudio con la población normal con baja vulnerabilidad, utilizamos los datos de un estudio previo de nuestro grupo en el que se validaron diferentes instrumentos de evaluación en población española (Borra, 2002), en el caso del BITE tomamos los datos de su validación original (Henderson y Freeman, 1987). Para seleccionar las personas con baja vulnerabilidad a tener un trastorno alimentario se seleccionaron a aquellas que puntuaron por debajo de 30 en el EAT-40 (Castro et al., 1991). En la Tabla 1 se presentan las medias y desviaciones típicas de la población general con baja vulnerabilidad a tener un trastorno alimentarios en los cuestionarios utilizados.

\section{Procedimiento}

Para obtener la muestra se realizó un submuestreo aleatorio. La asignación a las condiciones de tratamiento se ha realizado de forma aleatoria. La selección de la muestra, la evaluación de las pacientes, la asignación aleatoria a los grupos experimentales, la evaluación postratamiento y el análisis de los datos se realizó por personas ciegas al diseño experimental. Para ello se siguieron las recomendaciones CONSORT (Moher, Schulz y Altman, 2001). Este trabajo siguió todos principios éticos aprobados por el Comité Ético del Hospital.

Tabla 1. Medias y desviaciones típicas de la población normal con baja vulnerabilidad en los instrumentos utilizados.

\begin{tabular}{lll}
\hline Variable & $M(S D)$ & Fuente \\
\hline BIATQ & $7.1(28.59)$ & Borra (2002) \\
BAT & $25.83(13.94)$ & Borra (2002) \\
BASS & $29.60(5.33)$ & Borra (2002) \\
SIBID & $59.79(31.53)$ & Borra (2002) \\
BITE & $33(2.57)$ & Henderson y Freeman (1997) \\
EAT-40 & $9.73(5.68)$ & Borra (2002) \\
BSI & $35.46(26.50)$ & Borra (2002) \\
\hline
\end{tabular}

Nota: BIATQ= Body Image Automatic Thoughts Questionnaire; BAT= Body Attitude Test; BASS= Body Areas Satisfaction Scale; SIBID $=$ Situational Inventory of Body-Image Dysphoria; BITE $=$ Bulimic Investigatory Test Edinburgh; EAT= Eating Attitudes Test; BSI= Brief Symptom Inventory. 
La TCC para BN (Wilson et al., 1997) se realizó en grupo con un terapeuta experto en el tratamiento de los trastornos alimentarios y un coterapeuta. La frecuencia de las sesiones era semanal y con 2 horas de duración. Se utilizaron 19 sesiones de tratamiento. La TCC para la AN (Gardner et al., 1997) se realizó individualmente, con una frecuencia de 1 sesión semanal en la fase I de tratamiento y una sesión quincenal en las fases II y en la III. Se utilizó una media de 23 sesiones de tratamiento. El tratamiento fue siempre ambulatorio.

La TCC para la IC se aplicó en grupo y en 15 sesiones de tratamiento. El componente de realidad virtual se llevó a cabo de forma individual, una vez a la semana y la duración de las sesiones era de 1 hora. Se realizaron 8 sesiones de tratamiento (Marco et al., 2013)

Las pacientes no recibieron tratamiento farmacológico durante el tratamiento y a excepción de aquellas que ya lo tenían prescrito con anterioridad (un TCANE tomaba $\mathrm{Pa}$ roxetina y una AN Bromacepan), se mantuvo o redujo la dosis según los casos. El tratamiento fue siempre de carácter ambulatorio.

\section{Resultados}

Para realizar este trabajo fueron evaluadas 34 pacientes y asignadas aleatoriamente a las condiciones de tratamiento. En las primeras sesiones, cuatro participantes fueron excluidas del programa de tratamiento porque interferían gravemente en los grupos de terapia. Estas pacientes presentaban, además del trastorno alimentario, graves trastornos de la personalidad y depresión mayor. De las 30 pacientes que comenzaron el tratamiento, 11 pacientes abandonaron el tratamiento, 4 de la condición TTAIC y 7 en la condición TTAE. No se encontraron diferencias significativas entre ambas condiciones de tratamiento antes del mismo, lo cual se comprobó con prueba $t$ para muestras independientes. En el año de seguimiento una paciente abandonó la condición TTAE. La muestra final estuvo compuesta por 18 pacientes: 9 en la condición TTAE y 9 en la condición TTAIC.

En la Tabla 2 podemos ver como al finalizar el tratamiento se produce una mejoría en ambas condiciones de tratamiento, es decir todos los pacientes mejoran. Esta mejoría se produce en las medidas de IC, en las medidas de psicopatología de los trastornos alimentarios y en la medida de psicopatología general. Esta mejoría se incrementa en el seguimiento al año (Marco et al., 2013).

Tabla 2. Medias y desviaciones típicas de los participantes en los 3 momentos: pre, postratamiento y seguimiento.

\begin{tabular}{|c|c|c|c|c|c|c|c|c|c|c|c|c|}
\hline \multirow[b]{3}{*}{ Variable } & \multicolumn{4}{|c|}{ Pretratamiento } & \multicolumn{4}{|c|}{ Postratamiento } & \multicolumn{4}{|c|}{12 meses } \\
\hline & \multicolumn{2}{|c|}{ TTAIC } & \multicolumn{2}{|c|}{ TTAE } & \multicolumn{2}{|c|}{ TTAIC } & \multicolumn{2}{|c|}{ TTAE } & \multicolumn{2}{|c|}{ TTAIC } & \multicolumn{2}{|c|}{ TTAE } \\
\hline & $M$ & $S D$ & $M$ & $S D$ & $M$ & $S D$ & $M$ & $S D$ & $M$ & $S D$ & $M$ & $S D$ \\
\hline$\overline{\text { BIATQ }}$ & 78.9 & 21.4 & 64.5 & 30.3 & 7 & 39.6 & 49.4 & 42.4 & -5.4 & 21.6 & 33.6 & 43.6 \\
\hline BAT & 66.4 & 10 & 61.1 & 14.8 & 36.2 & 16.8 & 60.1 & 24.1 & 29.5 & 12 & 46.2 & 23.3 \\
\hline BASS & 19.8 & 2.7 & 21.7 & 4.8 & 27.5 & 4.3 & 24.8 & 5.8 & 30.1 & 2.10 & 23.7 & 5.1 \\
\hline SIBID & 147.1 & 22.8 & 138.2 & 23.3 & 83.5 & 41.9 & 118.7 & 46.2 & 64.4 & 37.7 & 103.7 & 50.5 \\
\hline BITE & 25.8 & 9.7 & 24.1 & 8.5 & 8.2 & 6.1 & 16.1 & 8.3 & 4.5 & 4.2 & 15.5 & 10.3 \\
\hline EAT-40 & 47.2 & 15.6 & 53.3 & 13.2 & 17 & 9.4 & 34.4 & 25.5 & 12 & 7.3 & 28.1 & 19.2 \\
\hline BSI & 84.7 & 28.9 & 102.4 & 23.2 & 34.2 & 24.8 & 76 & 40.7 & 39.6 & 25.9 & 67.6 & 41.8 \\
\hline
\end{tabular}

Nota: Adaptado de Marco et al.,(2013); TTAIC= Tratamiento Trastornos Alimentarios ampliado con intervención sobre la Imagen Corporal; TTAE: Tratamiento Trastornos Alimentarios Estándar; BIATQ= Body Image Automatic Thoughts Questionnaire; BAT= Body Attitude Test; BASS= Body Areas Satisfaction Scale; SIBID= Situational Inventory of Body-Image Dysphoria; BITE = The Bulimic Investigatory Test Edinburgh; EAT= Eating Attitudes Test; BSI $=$ Brief Symptom Inventory.

Cuando comparamos las puntuaciones obtenidas por las participantes con la población general con baja vulnerabilidad observamos que en la condición en la que se ha realizado una intervención específica sobre la IC (TTAIC) al finalizar el tratamiento las participantes presentan puntuaciones similares a la población general con baja vulnerabilidad a los trastornos alimentarios en las medidas de IC: en la frecuencia de pensamientos negativos automáticos que tienen las pacientes sobre la apariencia y el cuerpo (BIATQ) ( $t_{(105)}=$ .09 , NS) y de la satisfacción con determinadas áreas del cuerpo y el satisfacción corporal (BASS) $\left(t_{(105)}=.09\right.$, NS). Esta mejoría sigue evolucionando en el seguimiento al año en todas las medidas produciéndose la normalización en el malestar producido por situaciones relacionadas con el cuerpo $(\mathrm{SIBID})\left(t_{(105)}=.41, N S\right)$ y las actitudes hacia el cuerpo
$(\mathrm{BAT})\left(t_{(105)}=.77, \mathrm{NS}\right)$ en el seguimiento al año. Estos datos los podemos ver en la Tabla 3.

Las participantes que había sido tratadas en la condición TTAIC al finalizar el tratamiento presentaban puntuaciones similares a la población general con baja vulnerabilidad a los trastornos alimentarios en las medidas de psicopatología de los trastornos alimentarios, en el EAT-40 $\left(t_{(106)}=3.45, N S\right)$ y en el BITE $\left(t_{(37)}=3.57, p<.01\right)$ donde las participantes presentaban mejores puntuaciones que la población general. En el seguimiento al año las participantes mostraban puntuaciones similares a la población general con baja vulnerabilidad a los trastornos alimentarios en el EAT- 40 y en el BITE. En la misma línea que los resultados anteriores, podemos ver en la Tabla 3 que las pacientes de la condición TTAIC al finalizar el tratamiento y en el seguimiento al año presentaban puntuaciones similares a la población general con baja vulne- 
rabilidad a los trastornos alimentarios en las medidas de psicopatología secundaria, BSI $\left(_{(105)}=.13, \mathrm{NS} ; t_{(105)}=.45, \mathrm{NS}\right)$.

Tabla 3. Diferencias entre las puntuaciones de las participantes de la condición TTAIC con las de la población general con baja vulnerabilidad a los trastornos alimentarios al finalizar el tratamiento y en el seguimiento.

\begin{tabular}{lcccccccc}
\hline & \multicolumn{4}{c}{ Postratamiento } & \multicolumn{3}{c}{12 meses } \\
\cline { 2 - 9 } Variables & $t$ & $d f$ & $p<$ & $g^{\mathrm{a}}$ & $t$ & $d f$ & $p<$ & $g^{\mathrm{a}}$ \\
\hline BIATQ & .09 & 105 & $\mathrm{NS}$ & $\mathrm{a}$ & .74 & 105 & $\mathrm{NS}$ & $\mathrm{a}$ \\
BAT & 2.15 & 105 & .05 & .74 & .77 & 105 & $\mathrm{NS}$ & $\mathrm{a}$ \\
BASS & 1.14 & 105 & $\mathrm{NS}$ & $\mathrm{a}$ & .29 & 105 & $\mathrm{NS}$ & $\mathrm{a}$ \\
SIBID & 2.09 & 105 & .05 & .72 & .41 & 105 & $\mathrm{NS}$ & $\mathrm{a}$ \\
BITE & 3.57 & 37 & .001 & $1.30 \mathrm{~b}$ & 1.07 & 37 & $\mathrm{NS}$ & $\mathrm{a}$ \\
EAT-40 & 3.45 & 106 & $\mathrm{NS}$ & $\mathrm{a}$ & 1.12 & 106 & $\mathrm{NS}$ & $\mathrm{a}$ \\
BSI & .13 & 105 & $\mathrm{NS}$ & $\mathrm{a}$ & .45 & 105 & $\mathrm{NS}$ & $\mathrm{a}$ \\
\hline
\end{tabular}

Nota: TTAIC $=$ Tratamiento Trastornos Alimentarios ampliado con intervención sobre la Imagen Corporal; BIATQ= Body Image Automatic Thoughts Questionnaire; BAT $=$ Body Attitude Test; BASS= Body Areas Satisfaction Scale; SIBID= Situational Inventory of Body-Image Dysphoria; BITE $=$ The Bulimic Investigatory Test Edinburgh; EAT-40= Eating Attitudes Test; BSI= Brief Symptom Inventory. NS: no existen diferencias estadísticamente significativas.

a Hedge's $g$ solo se calcula cuando hay diferencias significativas; ${ }^{b}$ los participantes presentaban mejores puntuaciones que la población normal.

Por el contrario, en la condición donde no se ha intervenido específicamente en la IC, al finalizar el tratamiento y en el seguimiento al año, las participantes no presentan puntuaciones similares a la población general con baja vulnerabilidad a los trastornos alimentarios en las medidas de IC (BAT, BASS BIATQ y SIBID), ni en las medidas de psicopatología de los TA (BITE y EAT-40), ni en la medidas de psicopatología secundaria (IBS). En la Tabla 4 podemos ver la comparación entre las puntuaciones de las pacientes de la condición TTAE con las de la población general con baja vulnerabilidad a los TA.

Tabla 4. Diferencias entre las puntuaciones de las participantes de la condición TTAE con las de la población general con baja vulnerabilidad a los TA al finalizar el tratamiento y en el seguimiento.

\begin{tabular}{lcccccccc}
\hline & \multicolumn{3}{c}{ Postratamiento } & \multicolumn{4}{c}{12 meses } \\
\cline { 2 - 9 } Variables & $t$ & $d f$ & $p<$ & $g^{a}$ & $t$ & $d f$ & $p<$ & $g^{\text {a }}$ \\
\hline BIATQ & 4.06 & 105 & .001 & 1.40 & 2.54 & 105 & .01 & .87 \\
BAT & 7.21 & 105 & .001 & 2.44 & 4.17 & 105 & .001 & 1.41 \\
BASS & 2.55 & 105 & .01 & .88 & 3.16 & 105 & .001 & 1.09 \\
SIBID & 5.14 & 105 & .001 & 1.78 & 3.78 & 105 & .001 & 1.03 \\
BITE & 7.50 & 37 & .001 & 2.79 & 6.03 & 37 & .001 & 2.24 \\
EAT-40 & 7.98 & 106 & .001 & 2.75 & 6.94 & 106 & .001 & 2.40 \\
BSI & 3.57 & 105 & .001 & 1.02 & 3.30 & 105 & .001 & 1.14 \\
\hline Nota: TAE & & & & & & & &
\end{tabular}

Nota: TTAE $=$ Tratamiento Trastornos Alimentarios Estandard; BIATQ $=$ Body Image Automatic Thoughts Questionnaire; BAT $=$ Body Attitude Test; BASS $=$ Body Areas Satisfaction Scale; SIBID $=$ Situational Inventory of Body-Image Dysphoria; BITE= The Bulimic Investigatory Test Edinburgh; EAT-40= Eating Attitudes Test; BSI= Brief Symptom Inventory; NS: no existen diferencias estadísticamente significativas.

${ }^{a}$ Hedge's $g$ solo se calcula cuando hay diferencias significativas;

Estos resultados nos indican que las pacientes de la condición TTAIC al finalizar el tratamiento y en el seguimiento al año se había producido una mejoría clínicamente significativa en las medidas de IC, de psicopatología de los trastornos alimentarios y en la psicopatología secundaria ya que el cam- bio fue fiable y las puntuaciones obtenidas eran similares a las de la población general con baja vulnerabilidad. Por el contrario, en las pacientes de la condición TTAE al finalizar el tratamiento y en el seguimiento al año, no se produjo una mejoría clínicamente significativa, ni en las medidas de IC, ni en las de psicopatología específica de los trastornos alimentarios ni en la psicopatología secundaria.

\section{Discusión}

En las últimas décadas se ha utilizado el análisis del cambio clínicamente significativo para comprobar la eficacia del tratamiento diversos trastornos mentales (Blancard et al., 1990; Jacobson et al., 1984; Sheldrick et al., 2001). En el caso concreto de los trastornos alimentarios, existen muy pocos estudios donde se haya realizado un análisis de la eficacia clínica de los tratamientos aplicados. En un estudio previo (Marco et al., 2013) se demostró que la TCC para los TA, ampliado con un componente específico para la IC mediante técnicas de realidad virtual fue más eficaz que la TCC para los trastornos alimentarios. Esta mejoría fue mayor tanto desde un análisis de diferencias estadísticamente significativas como desde un análisis del cambio clínicamente significativo. Al finalizar el tratamiento y en el seguimiento al año solo la condición en la que se trató la IC, presentaban una reducción de la psicopatología del trastornos alimentarios y de la IC a niveles similares a la de la población general. Sin embargo, pensamos que en la evaluación de la eficacia de los tratamientos en los trastornos alimentarios hay que tener en cuenta que en la población general existe un descontento normativo (Rodin et al., 1984) y que un alto porcentaje de mujeres presentan actitudes, creencias y comportamientos relacionados con el cuerpo y la comida similares a los que presentan las pacientes diagnosticadas de AN y BN (Cash y Habrosky, 2004; Clarke y Palmer, 1983). Por este motivo para comprobar si un tratamiento ha producido una mejoría clínicamente significativa los resultados tendrían que ser comparados con la población general con baja vulnerabilidad a padecer un trastorno alimentario.

El principal objetivo de este trabajo fue comprobar si al añadir un componente de tratamiento centrado en la IC a la TCC para los trastornos alimentarios, produciría una mejoría mayor clínicamente significativa que cuando aplicamos la TCC estándar cuando comparamos los resultados del tratamiento con las personas con baja vulnerabilidad a tener un trastorno alimentario. En este trabajo hemos comprobado que al finalizar el tratamiento, en la condición que se ha tratado la IC, presentaban una reducción de la psicopatología de la IC, de los trastornos alimentarios y de la psicopatología general, similar a los niveles de la población general con baja vulnerabilidad. En la condición que no se ha intervenido específicamente en la IC no se ha producido una mejoría clínicamente significativa.

Los resultados obtenidos en este estudio ponen de manifiesto la conveniencia de incluir un componente de trata- 
miento que aborde las alteraciones de la IC en el protocolo de tratamiento general de los trastornos alimentarios ya que vemos que se produce una mejoría mayor en las actitudes, pensamientos, emociones y conductas relacionadas con el cuerpo y el aspecto físico. Teniendo en cuenta la importancia de la IC como factor mantenedor y de pronóstico en los trastornos alimentarios (Shisslak y Crago, 2001; Stice y Shaw, 2002) el hecho de obtener una mejoría y normalización es muy importante porque puede evitar futuras recaídas.

Estos resultados van en la línea de todos aquellos autores (Cash y Habrosky, 2004; Nye y Cash, 2006; Perpiñá et al., 2000) que plantean que para mejorar y normalizar los problemas de IC no basta con una intervención superficial, sino que es necesario un tratamiento específico que aborde todas y cada una de las dimensiones de las alteraciones de la IC (actitudinal, perceptiva, y emocional).

Actualmente algunos autores (Fairburn et al, 2009) están indicando y demostrando la necesidad de extender el tratamiento de los trastornos alimentarios hacia otros componentes como el perfeccionismo, los problemas interpersonales y la autoestima. Después de este trabajo consideramos necesario la intervención estructurada y amplia en la IC. De igual manera este trabajo apoya la idea de que es conveniente que la TCC para los trastornos alimentarios abordara no solo los síntomas y signos actuales mantenedores del trastorno alimentario, sino que se extendiera hacia factores etiológicos que están en la base del trastorno alimentario (Fairburn et al, 2003), como son las alteraciones de la IC.

Existen pocos trabajos hasta la fecha en el que se haga un estudio controlado para comprobar si es beneficioso realizar una intervención específica y estructurada sobre las IC en pacientes con trastornos alimentarios (Farrell et al., 2006). En este trabajo se ha realizado una intervención estructurada y controlada desde el inicio del tratamiento hasta el seguimiento, lo cual nos permite controlar variables externas como pueden ser tratamientos externos, cambios vitales, etc. Una de las fortalezas de este trabajo es la muestra utilizada proveniente de un servicio de Salud Mental público, pacientes diagnosticadas de AN, BN y TCANE. Los criterios de inclusión eran muy amplios por lo que podemos afirmar que la muestra es representativa de las pacientes atendidas en la práctica clínica diaria (Bell, 2001; Haas y Clopton, 2003; Mahon, 2000). Además, el tamaño de la muestra es similar al

\section{Referencias}

American Psychiatric Association. (2000). Diagnostic and statistical manual of mental disorders (4th ed, text rev.). Washington, DC: APA.

Blanchard, E. B., Appelbaum, K. A., Radnitz, C. L., Michultak, D., Morrill, B., Kirsch, C., ... Dentinger, M. P. (1990). Placebo controlled evaluation of abbreviated progressive muscle relaxation and of relaxation combined with cognitive therapy in the treatment of tension headache. Journal of Consulting and Clinical Psychology, 58, 210-215.

Bell, L. (2001). What predicts failure to engage in or drop out from treatment for bulimia nervosa an what implications does this have for treatment? Clinical Psychology and Psychotherapy, 8, 424-435. encontrado en otros trabajos con muestra clínicas en los trastornos alimentarios (Nye y Cash, 2006). El mostrar el seguimiento al año, también nos permite comprobar que los resultados son consistentes y que siguen evolucionando en la línea esperada, aspecto muy importante dado las tasas de recaídas de los TA.

Una de las limitaciones de este diseño es que los participantes de la condición TTAIC recibieron más sesiones durante el tratamiento, sin embargo, como hemos comprobado en un trabajo previo (Marco et al., 2013) en los estudios sobre eficacia de la tratamientos se ha demostrado que en trastornos tan complejos como los trastornos alimentarios, lo importante no es el número de sesiones si no el contenido de las sesiones. Otra de las limitaciones son los abandonos ocurridos durante el tratamiento. No obstante, hay que indicar que en la condición TTAIC se han producido la mitad de abandonos que en la condición TTAE. Este efecto de adherencia al tratamiento lo hemos podido comprobar en otras investigaciones en las que se ha utilizado nuevas tecnologías como la realidad virtual (Perpiñá, Marco, Botella y Baños, 2004; Riva, Bachetta, Baruffi y Molinari, 2002). Otra limitación de este estudio es que la exclusión de pacientes con graves trastornos de la personalidad se realizó una vez se había empezado el tratamiento, por este motivo las condiciones no tienen el mismo número de participantes. Cuando trabajos con personas con graves trastornos de la personalidad, en ocasiones, no descubrimos comportamientos y actitudes que interfieren con la terapia hasta que ha comenzado (Lineham, 1993). En relación con lo anterior, es bien conocida, la dificultad que conlleva aplicar tratamientos estructurados y homogéneos a las personas diagnosticadas de trastornos alimentarios y la dificultad de trabajar los aspectos de la IC tal y como nosotros lo hemos realizado. Prueba de ello, como señala Nye y Cash (2006) es que hasta la fecha, existen pocos trabajos en el que se analice diferencialmente el hecho de incorporar un componente de IC al tratamiento convencional de los TA.

Agradecimientos.- Este trabajo ha sido parcialmente subvencionado por el ministerio de Sanidad y Consumo (FIS) (99/0997), por el Hospital Provincial de Castellón y por CIBER. CIBER Fisiopatología de la Obesidad y Nutrición.

Borra, C. (2002). Validación del cuestionario sobre los pensamientos automáticos a cerca de la Imagen Corporal, BLATQ (Trabajo de investigación no publicado). Valencia: Universidad de Valencia.

Bully, P., Elosua, P. y López-Jáuregui, A. (2012). Insatisfacción corporal en la adolescencia: Evolución en una década. Anales de Psicología, 28 (1), 196-202.

Butters, J. W. y Cash, T. F. (1987). Cognitive-behavioral treatment of women's body-image dissatisfaction. Journal of Consulting and Clinical Psychology, 55, 889-897.

Carter, P. I. y Moss, R. A. (1984). Screening for anorexia and bulimia nervosa in a college population: Problems and limitations. Addictive Behaviors, 9, 417-419. 
Cash, T. F. (1991). Body Image therapy: a program for self directed change. New York: Guilford Press.

Cash, T. F. (1994). The situacional inventory of body- image dysphoria: Contextual assessment of a negative body image. The Behavior Therapist, 17, 133-134.

Cash, T. F. y Grant, J. R. (1996). Cognitive-behavioral treatment of bodyimage disturbances. En M. Hersen, y V. Van Hesselt (Eds.), Sourcebook of psychological treatment manuals for adult disorders (pp. 567-614). NewYork: Plenum Press.

Cash, T. F. y Hrabosky, J. I. (2004). Treatment of body image disturbances. En J. K. Thompson (Ed.), Handbook of eating disorders and obesity (pp. 515-541). Hoboken, NJ: Wiley.

Cash, T. F., Lewis, R. J. y Keeton, P. (1987). Development and validation of the Body Image Automatic Thoughts Questionnaire. Trabajo presentado en el Annual Meeting of the Southeastern Psychological Association, Atlanta, GA.

Castro, J., Toro, J., Salamero, M. y Guimerá, E.(1991) .The Eating Attitudes Test: validation of the Spanish version. Psychological Assessment, 7, 175190.

Christensen, L. y Mendoza, J. L. (1986). A method of assessing change in a single subject: An alteration of the RC Index. Behavior Therapy, 17, 305308.

Clarke. M. G., y Palmer, R. L. (1983) Eating attitudes and neurotic symptoms in university students. The British Journal of Psychiatry, 142, 299-304.

Derogatis, L.R. y Melisaratos, N. (1983). The Brief Symptom Inventory: An introductory report. Psychological Medicine, 13, 595- 605.

Fairburn, C. G. (2002). Cognitive-behavioral therapy for bulimia nervosa. En C. G. Fairburn, y K. D. Brownell (Eds.), Eating disorders and obesity: A comprehensive handbook (pp. 233-237). New York: Guilford.

Fairburn, C. G., Cooper, Z., Doll, H. A., O’Connor, M. E., Bohn, K., Hawker, D. M., ... y Palmer, R. L. (2009). Transdiagnostic cognitivebehavioral therapy for patients with eating disorders: a two-site trial with 60-week follow-up. The American journal of psychiatry, 166(3), 311.319.

Fairburn, C. G., Cooper, Z. y Shafran, R. (2003). Cognitive behavior therapy for eating disorders: A 'transdiagnostic' theory and treatment. Behavior Research and Therapy, 41, 509-528.

Fairburn, C. G., Marcus, M. D. y Wilson, G. T. (1993). Cognitive-behavioral therapy for binge eating and bulimia nervosa: A comprehensive treatment manual. En C. G. Fairburn, y G. T. Wilson (Eds.), Binge eating: Nature, assessment, and treatment. New York: Guilford Press.

Fairburn, C. G., Peveler, R. C., Jones, R., Hope, R. A. y Doll, H. A. (1993). Predictors of 12-month outcome in bulimia nervosa and the influence of attitudes to shape and weight. Journal of Consulting and Clinical Psychology, 61, 696-698.

Farrell, C., Shafran, R. y Lee, M. (2006). Empirically evaluated treatments for body image disturbance: A review. European Eating Disorders Review, 14, 289-300.

First, M. B., Gibbon, M., Spitzer, R. L., Williams, J. B. W. y Benjamin, L.S. (1997). Structured Clinical Interview for DSM-IV Axis II Personality Disorders, (SCID-II). Washington, D.C.: American Psychiatric Press, Inc.

First, M. B., Gibbon, M., Spitzer, R. L., Williams, J. W. y Benjamin, L. S. (1999). Entrevista clínica estructurada para los trastornos de la personalidad del eje II del DSM-IV-TR.Barcelona: Masson.

First, M. B., Spitzer, R. L., Gibbon, M. y Williams, J. B. W. (2002). Structured Clinical Interview for DSM-IV-TR Axis I Disorders, Research Version, Nonpatient Edition. (SCID-I/NP) .New York: Biometrics Research, New York State Psychiatric Institute.

Follette, W. C. y Callaghan, G. M. (2001). The evolution of clinical significance. Clinical Psychology: Science and Practice, 8(4), 431-435.

Garner, D. M. (2002). Body image and anorexia nervosa. En T. F. Cash, y T. Pruzinsky, (Eds.), Body image: A handbook of theory, research, and clinical practice (pp. 295-303). New York: Guilford.

Garner, D. M. y Garfinkel, P. E. (1979). The Eating Attitudes Test: An index of the symptoms of anorexia nervosa. Psychology and Medicine, 9, 273279.

Garner, D. M., Olmstead, M. P., Bohr, Y. y Garfinkel, P. E. (1982). The eating attitudes test: Psychometric features and clinical correlates. Psychological Medicine, 12, 871-878.
Garner, D. M., Vitousek K. M. y Pike K. M. (1997).Cognitive behavioral therapy for anorexia nervosa. En Garner, D. M., y Garfinkel, P. E. (Eds), Handbook of treatment for eating disorders. New York:Guilford Press.

Gila, A., Castro, J., Gómez, M. J., Toro, J. y Salamero, M. L. (1999). The body attitudes test: Validation of the Spanish version. Eating and Weight Disorders ,4, 175-178.

Gleaves, D. H., Williamson, D. A. y Barker, S. E. (1993). Confirmatory factor analysis of a multidimensional model of bulimia nervosa. Journal of Abnormal Psychology,

102, 173-176.

Hass, H. L. y Clopton, J. R. (2003). Comparing clinical and research treatments for eating disorders. International Journal of Eating Disorders, 33,412420.

Hedges, L. V. y Olkin, I. (1985). Statistical Methods for Meta-Analysis. New York: Academic Press.

Henderson, M. y Freeman, C. (1987). A self-rating scale for bulimia: the BITE. British Journal of Psychiatry, 150, 18-24.

Jacobson, N. S., Follette, W. C. y Revenstorf, D. (1986). Toward a standard definition of clinically significant change. Behavior Therapy, 17, 308-311.

Jacobson, N. S., Follette, W. C., Revenstorf, D., Baucom, D. H., Hahlweg, K., y Margolin, G. (1984). Variability in outcome and clinical significance of behavioral marital therapy: A reanalysis of outcome data. Journal of Consulting and Clinical Psychology, 52(4), 497-504.

Jacobson, N. S. y Revenstorf, D. (1988). Statistics for assessing the clinical significance of psychotherapy techniques: Issues, problems, and new developments. Behavioral Assessment, 10, 133-145.

Jacobson, N. S. y Truax, P. (1991). Clinical Significance: a stadistical approach to defining meaningful change in psychotherapy research. Journal of Consulting and Clinical Psychology, 59, 1, 12-19.

Kendall, P. C., Marrs-Garcia, A., Nath, S. R. y Sheldrick, R. C. (1999). Normative Comparisons for the evaluation of clinical significance. Journal of Consulting and Clinical Psychology, 53, 43-48.

Linehan, M. M., (1993). Cognitive-behavioral treatment of borderline personality disorder. New York: Guilford Press.

Lobbestael, J., Leurgans, M., y Arntz, A. (2011). Inter-rater reliability of the Structured Clinical Interview for DSM-IV Axis I Disorders (SCID I) and Axis II Disorders (SCID II). Clinical psychology \& psychotherapy, 18, 75-79.

Lundgren D. J., Danoff-Burg S., y Anderson D. A. (2004). Cogntitvebehavioral therapy for bulimia nervosa: an empirical analysis of clinical significance. International Journal of Eating Disorders, 35, 262-274.

Mahon, J. (2000). Dropping out from psychological treatment for eating disorders: What are the Issues? European Eating Disorders Review, 8, 198216.

Marco, J. H., Perpiñá, C. y Botella, C. (2013). Effectiveness of cognitive behavioural therapy supported by virtual reality in the treatment of body image in eating disorders: one year follow-up. Psychiatry Research, 209(3), 619-625,

Moher, D., Schulz, K. F. y Altman, D. G., (2001). The CONSORT statement: revised recommendations for improving the quality of reports of parallel group randomised trials. The CONSORT Group. The Lancet, 357, 1191-1194.

National Institute for Clinical Excellence. (2004). Eating Disorders: Core interventions in the treatment and management of anorexia nervosa, bulimia nervosa and related eating disorders. Nice Clinical Guideline. No. 9. Recuperado de http://www.nice.org.uk

Nye, S. y Cash, T. F. (2006). Outcome of manualized cognitive-behavioral body image therapy with eating disordered women treated in a private clinical practice. Eating disorders, 14, 31-40.

Ogles, B. M., Lunnen, K. M. y Bonesteel, K. (2001). Clinical significance: History, application, and current practice. Clinical Psychology Review, 21(3), 421-446.

Perpiñá, C., Botella, C. y Baños, R. M. (2000). Imagen corporal en los trastornos alimentarios. Evaluación y tratamiento por medio de realidad virtual. Valencia: Promolibro.

Perpiñá, C., Botella, C. y Baños, R. M. (2003). Virtual reality in eating disorders. European Eating Disorders Review, 11, 261-278.

Perpiñá, C., Borra, C., Baños, R. Botella, C. Quero, S. y Jorquera, M. (2003). Psychometric properties of the Body Image Automatic Thoughts Questionnaire (BIATQ) in a Spanish population. Poster presentado en 37th Annual Conven- 
tion of Association for Advancement of Behavior Therapy . Boston (EEUU).

Perpiñá, C., Marco, J. H., Botella, C. y Baños, R. (2004). Tratamiento de la Imagen Corporal en los trastornos alimentarios mediante tratamiento cognitivo-comportamental apoyado con realidad virtual: resultados al año de seguimiento. Psicología Conductual, 12 (3), 519-537.

Perpiña, C., Gallego, M. J. y Botella, C., (2006). Psychometric properties of the Situational Inventory of Body-Image Dysphoria-Short form in a Spanish sample. Body Image, 3, 301-306.

Probst, M., Vandereycken, W., Van Coppenolle, H. y Vanderlinden, J. (1995). The Body Attitude Test for patients with an eating disorder: Psychometric characteristics of a new questionnaire. Eating Disorders: The Journal of Treatment and Prevention, 3, 133- 145.

Riva, G., Bacchetta, M., Baruffi, M. y Molinari, E. (2002). Virtual-realitybased multidimensional therapy for the treatment of body image disturbances in binge eating disorders: A preliminary controlled study. IEEE Transactions on Information Technology in Biomedicine, 6, 224-234.

Rodin, J., Silberstein, L. \& Striegel-Moore, R. (1984). Women and weight: a normative discontent. Nebraska symposium on motivation. University of Nebraska Press.

Ruipérez, M. A., Ibañez, M. I., Lorente, E., Moro, M. y Ortet, G., (2001). Psychometric properties of the Spanish version of the BSI. European Journal of Psychological Assessment, 3, 241-250.

Schwartz, M. D. y Brownell, K. D., (2004). Obesity and body image. Body Image, 1, 43-56.

Sheldrick, R. C., Kendall, P. C. y Heimberg, R. G. (2001). The clinical significance of treatments: a comparison of three treatments for conduct disordered children. Clinical Psychology: Science and Practice, 8, 418-430.

Shisslak, C. M. y Crago, M. (2001). Risk and protective factors in the development of eating disorders. En J. K. Thompson y L. Smolak (Eds.),
Body image, eating disorders, and obesity in youth: Assessment, prevention, and treatment (pp. 103-125). Washington, DC: American Psychological Association.

Stice, E. (2002). Body image and bulimia nervosa. En T. F. Cash y T. Pruzinsky (Eds.), Body image: A bandbook of theory, research, and clinical practice(pp. 304-311). New York: Guilford.

Stice, E. y Shaw, H. E. (2002). Role of body dissatisfaction in the onset and maintenance of eating pathology: A synthesis of research findings. Jour nal of Psychosomatic Research, 53, 985-993.

Thompson, J. K. (1992). Body image: extent of disturbance, asociated features, theoretical models, assessment methodologies, intervention strategies, and a proposal for a new DSM-IV category-Body Image Disorder. En M. Hersen, R. M. Esisler y P. M. Miller (Eds.). Progress in Behav ior Modification (pp. 3-54). Sycamore, IL: Sycamore Press.

Thompson, J. K., Heimberg, L. J. y Clarke, A. J. (1996). Treatment of body image disturbances in eating disorders. En J. K. Thompson (Ed.), Body image, eating disorders, and obesity. Washington, DC: American Psychological Association.

Vitousek, K. B. (2002). Cognitive-behavioral therapy for anorexia nervosa. En C. G. Fairburn y K. D. Brownell (Eds.), Eating disorders and obesity: A comprehensive

bandbook (2nd ed.) (pp. 233-237). New York: Guilford Press.

Wilson, T. G., Fairburn, C. G. y Agras, W. S. (1997). Cognitive-behavioral therapy for bulimia nervosa. En D. M. Garner y P. E. Garfinkel (Eds.), Handbook of treatment for eating disorders (pp 67-93). New York: Guilford Press.

(Articulo recibido: 18-4-2012; revisado: 21-5-2013; aceptado: 24-1-2014) 